\title{
Cardiotrophin-1 is not associated with carotid or coronary disease and is inversely associated with obesity in patients undergoing coronary angiography
}

Umberto Vespasiani-Gentilucci ${ }^{1}$, Antonio De Vincentis ${ }^{1}$, Josepmaria Argemi ${ }^{2}$, Giovanni Galati ${ }^{1}$, Eduardo Ansò르. Giuseppe Patti ${ }^{3}$, Antonio Picardi ${ }^{1}$

${ }^{1}$ Campus Bio-Medico of University, Clinical Medicine - Hepatology, Rome, Italy 2University of Navarra, Pamplona, Italy

${ }^{3}$ Campus Bio-Medico University, Cardiology, Rome, Italy

Submitted: 16 November 2012

Accepted: 27 January 2013

Arch Med Sci 2013; 9, 4: 635-639

DOI: 10.5114/aoms.2013.37272

Copyright (c) 2013 Termedia \& Banach
Corresponding author: Dr. Antonio Picardi University Campus Bio-Medico of Rome Via A. del Portillo 200 00128 Rome, Italy Phone: +3906225411207 Fax: +390622541456 E-mail:

a.picardi@unicampus.it

\begin{abstract}
Introduction: Cardiotrophin-1 (CT-1) is a member of the interleukin-6 superfamily with known hypertrophic and protective actions upon cardiac myocytes. Although its effects on myocardial tissue and its role in hypertensive heart disease are well documented, there are no studies on CT-1 blood levels in patients with coronary artery disease. In this study we aimed to verify the relationships of serum CT-1 with vascular disease and metabolic parameters in a population of patients undergoing coronary angiography due to clinical indications.

Material and methods: Serum levels of CT-1 were investigated in a cohort of 81 consecutive patients (median age 68 years (95\% Cl: 64-71), 59 males) undergoing coronary angiography and carotid Doppler ultrasound. Exclusion criteria were: acute coronary syndrome, already-established ischemic cardiopathy, chronic inflammatory diseases and presence or past history of cancer.

Results: Levels of CT-1 were inversely correlated with body mass index (BMI) and waist circumference (WC) $(\rho=-0.261, p=0.02 ; \rho=-0.224, p=0.05$, respectively). Moreover, obese patients showed significantly lower CT-1 concentrations than non-obese ones (1.18 (0.64-1.64) ng/ml vs. $1.56(1.37-2.04) \mathrm{ng} / \mathrm{ml}, p=0.013)$, and serum CT-1 was significantly reduced in patients with elevated compared to those with normal WC (1.43 (0.94-1.60) ng/ml vs. $1.64(1.39-2.49) \mathrm{ng} / \mathrm{ml}$, $p=0.047)$. Concentrations of CT-1 did not correlate either with the other parameters of metabolic syndrome or with markers of cardiovascular disease (carotid intima-media thickness, presence of carotid or coronary artery plaques).

Conclusions: Our results failed to demonstrate any association between CT-1 and carotid or coronary disease. The inverse association with BMI and WC fits with the latest experimental data on the role of CT-1 in dysmetabolic conditions and could help to further clarify the role of CT-1 in obesity and diabetes.
\end{abstract}

Key words: metabolic syndrome, coronary artery disease, carotid artery disease.

\section{Introduction}

Cardiotrophin-1 (CT-1) is a member of the interleukin-6 (IL-6) superfamily, which shares the transmembrane signaling protein glycoprotein (gp) 130 as a receptor. It was originally identified as a factor that could induce hypertrophy on cardiac myocytes in vitro [1], but it has been shown to be widely expressed in various adult human tissues including muscle, heart, liver and adipose tissue [2]. To date, CT-1 has been mainly studied for its effects on myocardial tissue and its role in hypertensive heart dis- 
ease is well documented [3]. Designed primarily to function as a cardiomyocyte survival factor, chronic and excessive activation of CT-1 signaling has been postulated to be harmful, contributing to pathologic left ventricular hypertrophy and myocardial fibrosis [4].

Recently, growing interest has been focused on CT-1's role in adipocyte metabolism and atherogenic conditions, since other gp-130 receptor ligands, such as ciliary neurotrophic factor (CNTF) and interleukin-6 (IL-6), have been shown to affect body weight regulation and glucose tolerance [5-9]. The recent milestone study by Moreno-Aliaga et al. showed that mice lacking CT-1 develop a sort of metabolic syndrome (MS), characterized by adult-onset obesity, hyperglycemia, hyperinsulinemia and dyslipidemia, mainly due to insulin resistance and impaired fat mobilization, and evidenced the anti-obesity properties of chronic recombinant CT-1 (rCT-1) administration not only in $\mathrm{CT}-1^{-/-}$mice, but also in other models of obese mice, proposing a possible therapeutic use of rCT-1 in obesity and type 2 diabetes [10]. However, an apparent contradiction is raised by the attempt to fit these experimental results with previous studies on CT-1 levels in humans, which, conversely, reported that MS, obesity and hypertension are associated with elevated CT-1 concentrations [11-13].

In contrast with the amount of experimental and clinical literature on CT-1 in hypertensive heart disease, there are no studies on CT-1 blood levels in patients with coronary artery disease. Therefore, based on its effects on both cardiac muscle and metabolism, in the present study we aimed to verify the relationships of serum CT-1 with vascular disease and metabolic parameters in a population of patients undergoing coronary angiography due to clinical indications.

\section{Material and methods}

Serum levels of CT-1 were investigated in a cohort of 81 consecutive patients undergoing coronary angiography due to clinical indications in our Cardiology Unit between October 2010 and April 2011. All patients met the AHA/ACC class I indication for coronarography [14]: 30 subjects (37\%) had high risk criteria on non-invasive cardiovascular tests, in absence of symptoms, 21 (26\%) had stable symptoms of ischemic heart disease and high risk criteria on noninvasive cardiovascular tests, and 30 (37\%) had symptoms of incipient congestive heart failure (CHF) with angina and/or non-invasive tests suggestive of reversible myocardial ischemia. Patients with acute coronary syndrome, already-established ischemic cardiopathy (documented by previous coronarography or with regional wall motion abnormalities), chronic inflammatory diseases and presence or past history of cancer were excluded.
Anthropometric and biochemical parameters were measured, and each patient underwent carotid Doppler ultrasound for evaluation of carotid artery intima-media thickness (cIMT) and detection of atherosclerotic plaques. All blood samples were drawn after $8 \mathrm{~h}$ of fasting. Body mass index (BMI) was calculated using the following formula: weight (kg)/ height $^{2}(\mathrm{~m})$. Carotid artery intima-media thickness was computed as the maximum value from determinations in three tracts (common carotid, at one centimeter from the bifurcation and in the internal carotid artery), and carotid atherosclerotic plaque was defined as a focal thickening greater than $2 \mathrm{~mm}$. Coronary artery disease was defined as the presence of at least $50 \%$ stenosis in at least one major coronary artery. Serum CT-1 was determined using a home-made ELISA technique, essentially not different from that described by Moreno-Aliaga et al. [10]. Briefly, a 96-well plate was coated with monoclonal antibody MAB438 (R\&D, Illinois, USA) at $0.2 \mu \mathrm{g} /$ well in $100 \mu \mathrm{l}$ of phosphate buffer saline (PBS at $\mathrm{pH} 7.4$ ) for $18 \mathrm{~h}$ at $4^{\circ} \mathrm{C}$. After washing, the plate was blocked for $2 \mathrm{~h}$ at $37^{\circ} \mathrm{C}$ with PBS containing $0.1 \%$ casein and rinsed twice with PBS containing 0.05\% tween-20 (PBST). Serum samples mixed in sample diluent ( $0.5 \%$ tween- 80 and $1 \%$ BSA) were then added. After overnight incubation at $4^{\circ} \mathrm{C}$, plates were rinsed three times with PBST and incubated with a biotin-labeled rabbit secondary polyclonal antibody against human CT-1 (generated in our laboratory by injecting rabbits with recombinant $\mathrm{CT}-1$ ) diluted $1: 2000$ in PBST for $2 \mathrm{~h}$ at $37^{\circ} \mathrm{C}$. The plate was then rinsed three times with PBST and incubated for $30 \mathrm{~min}$ at room temperature with $100 \mu \mathrm{l} /$ well of 1 : 5000 diluted streptavidin-peroxidase complex (PIERCE, Illinois, USA). Finally, plates were washed five times with PBST and developed with TMB substrate. After stopping the reaction with sulfuric acid, absorbance was measured at $450 \mathrm{~nm}$.

\section{Statistical analysis}

Data are expressed as median $(95 \% \mathrm{Cl})$ or number and frequency (\%), as appropriate. Mann-Whitney $U$-test was used to compare independent variables between two groups and correlations were carried out by Spearman's rank correlation. Statistical significance was assumed at $p<0.05$. Statistical analyses were performed by using SPSS software (version 17.00; SPSS Inc., Chicago, IL, USA).

\section{Results}

The main features of the study group are listed in Table I. Median age was over 65 years old, with a clear prevalence of males (73\%). Median BMI was in the range of overweight, and there were high prevalences of diabetes, hypertension, dyslipidemia and MS, as expectable in a population of candidates 
for coronary angiography. Vascular complications, i.e. myointimal carotid thickening, carotid plaques and coronary disease, were evidenced in percentages fluctuating around $50 \%$.

While not significantly different between patients with and without MS (Table II), CT-1 levels were inversely correlated with body mass index (BMI) $(\rho=-0.26, p=0.02)$, and obese patients (BMI $\geq 30 \mathrm{~kg} / \mathrm{m}^{2}$ ) showed significantly lower CT-1 concentrations than non-obese ones (Table II and Figure 1). Accordingly, CT-1 levels were inversely correlated with waist circumference (WC) $(\rho=-0.224$, $p=0.046)$, and serum CT-1 was significantly reduced in patients with elevated WC $(>102 \mathrm{~cm}$ in men and $>88 \mathrm{~cm}$ in women) compared to those with normal WC according to the criteria of the National Cholesterol Education Program Adult Treatment Panel III guidelines [15] (Table II and Figure 1). However, CT-1 concentrations were not associated with the other parameters of MS (diabetes, hypertension, HDL cholesterol, triglycerides), nor with vascular disease (cIMT, presence of carotid or coronary artery plaques) (Table II). Furthermore, CT-1 levels were unrelated to clinical symptoms at presentation (no symptoms, stable angina or symptoms of incipient (HF) and no differences were observed between subjects with different indications for coronary angiography (data not shown).

\section{Discussion}

The present results, obtained in a population of candidates for coronary angiography, fail to demonstrate any association between CT-1 serum levels and carotid or coronary disease, while they are the first to find an inverse association between CT-1 circulating concentrations, and whole body and $a b$ dominal obesity in humans.

Cardiotrophin-1 is a member of the gp130 family of cytokines which has been widely studied for
Table I. Epidemiologic, anthropometric and metabolic features of the study group

\begin{tabular}{|c|c|}
\hline Variable & Results \\
\hline Age [years] & $68(64-71)$ \\
\hline $\operatorname{Sex}(M / F)$ & $59 / 22$ \\
\hline Body mass index $\left[\mathrm{kg} / \mathrm{m}^{2}\right]$ & $27.4(26.7,28.4)$ \\
\hline Waist circumference $[\mathrm{cm}]$ & $101(98-103)$ \\
\hline Glucose [mg/dl] & $97(94-103)$ \\
\hline Diabetes & $25(30.9 \%)$ \\
\hline Hypertension & $66(81.5 \%)$ \\
\hline $\begin{array}{l}\text { Hypo-HDL (<40 mg/dl in men } \\
\text { or }<50 \mathrm{mg} / \mathrm{dl} \text { in women })\end{array}$ & $41(50.6 \%)$ \\
\hline $\begin{array}{l}\text { Hypertriglyceridemia } \\
\text { (>150 mg/dl or treated) }\end{array}$ & $25(30.9 \%)$ \\
\hline Metabolic syndrome & $36(44.4 \%)$ \\
\hline Cardiotrophin-1 [ng/ml] & $1.5(1.35-1.65)$ \\
\hline Intima-media thickness, > $1.1 \mathrm{~mm}$ & $34(42 \%)$ \\
\hline $\begin{array}{l}\text { Carotid artery disease, presence } \\
\text { of plaques }\end{array}$ & $48(59.3 \%)$ \\
\hline $\begin{array}{l}\text { Coronary artery disease, } \\
\text { mono or multivessel }\end{array}$ & $39(48.1 \%)$ \\
\hline
\end{tabular}

Data reported as median (95\% Cl) or number and frequency (\%)

its effects on myocardial cells and its possible role in cardiac pathophysiology [2, 12]. Cardiotrophin-1 is produced by cardiomyocytes and non-cardiomyocytes in situations of biomechanical stress and activates different signaling pathways leading to cardiomyocyte hypertrophy as well as to myocardial fibrosis [3]. Actually, elevated blood CT-1 concentrations have been found in hypertensive patients $[12,16]$, higher in those with left ventricular hypertrophy and dysfunction [16, 17], and progressively increasing along with progression of heart failure stages [16].

Table II. CT-1 serum concentrations according to the metabolic or vascular diagnosis of interest

\begin{tabular}{|c|c|c|c|}
\hline \multirow[t]{2}{*}{ Variable } & \multicolumn{2}{|c|}{ CT-1 concentrations $[\mathrm{ng} / \mathrm{ml}]$} & \multirow[t]{2}{*}{ Value of $p$} \\
\hline & Yes & No & \\
\hline Obesity (BMI $\geq 30$ kg/m²) & $1.18(0.64-1.64)$ & $1.56(1.37-2.04)$ & 0.013 \\
\hline Elevated WC (> $102 \mathrm{~cm}$ in men and $>88 \mathrm{~cm}$ in women) & $1.43(0.94-1.60)$ & $1.64(1.39-2.49)$ & 0.047 \\
\hline Diabetes & $1.45(1.25-1.77)$ & $1.60(1.30-2.02)$ & 0.870 \\
\hline Hypertension & $1.64(1.35-3.41)$ & $1.49(1.23-1.72)$ & 0.187 \\
\hline Hypo-HDL (< 40 mg/dl in men or $<50$ mg/dl in women) & $1.41(0.98-1.54)$ & $1.65(1.37-2.34)$ & 0.069 \\
\hline Hypertriglyceridemia (> 150 mg/dl or treated) & $1.48(1.33-1.65)$ & $1.60(1.23-2.40)$ & 0.516 \\
\hline Metabolic syndrome & $1.55(1.21-2.01)$ & $1.47(1.35-1.92)$ & 0.78 \\
\hline Intima-media thickness, > $1.1 \mathrm{~mm}$ & $1.56(1.33-2.50)$ & $1.45(1.15-1.77)$ & 0.270 \\
\hline Carotid artery disease, presence of plaques & $1.48(1.02-2.40)$ & $1.51(1.35-1.72)$ & 0.996 \\
\hline Coronary artery disease, mono or multivessel & $1.55(1.33-1.92)$ & $1.46(1.23-2.02)$ & 0.737 \\
\hline
\end{tabular}


A

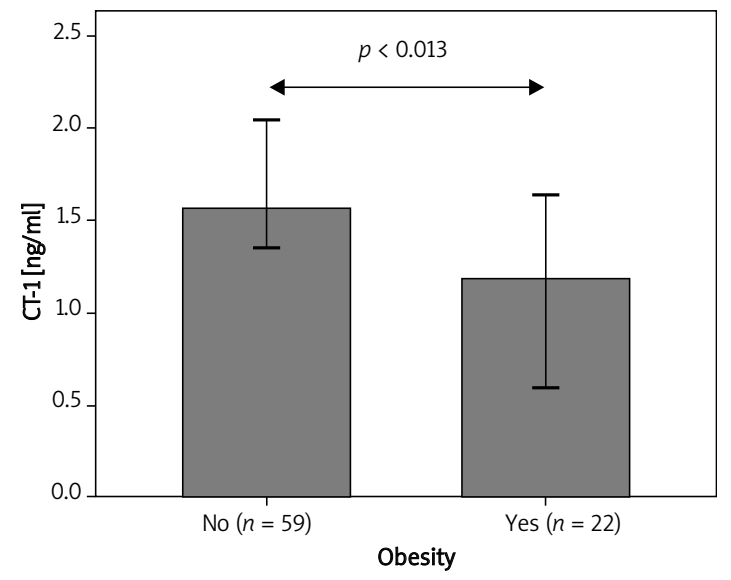

B

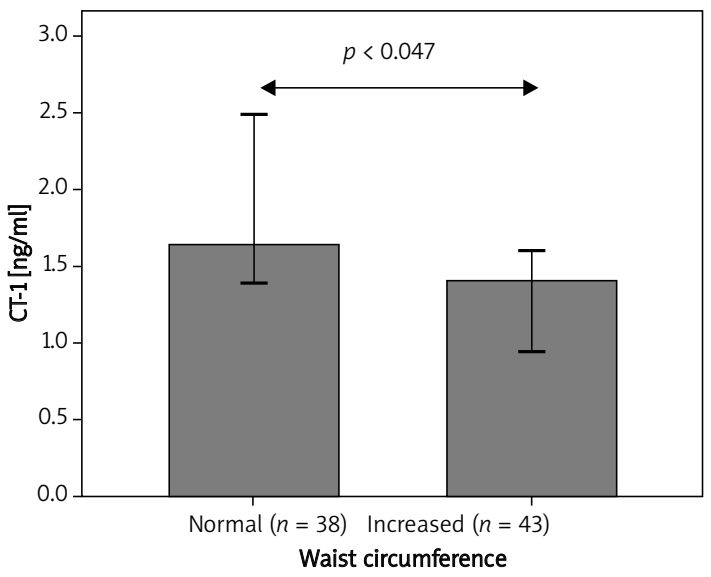

Figure 1. Reduced CT-1 level in patients with obesity (A) and increased waist circumference (B). Data are reported as median, error bars represent $95 \% \mathrm{Cl}$

Recent evidence has demonstrated that gp130 receptor ligands, such as CNTF and IL-6, can affect the balance between calorie procurement and disposal [5-9]. Ciliary neurotrophic factor can induce weight loss by effects on hypothalamic control of energy balance [18], and it has been proposed as a potential treatment for obesity and diabetes [19]. The effects of IL- 6 on energy metabolism remain controversial, and the discovery that IL-6 is produced and released from skeletal muscle during exercise [20] has challenged the classical view that it is a proinflammatory cytokine inducing insulin resistance and diabetes [8]. Most of CT-1's metabolic properties have been recently described by the study of Moreno-Aliaga et al., who demonstrated that $\mathrm{CT}-1^{-1-}$ mice develop mature-onset obesity, insulin resistance, and hypercholesterolemia despite reduced calorie intake, and that treatment with $\mathrm{rCT}-1$ reduces adiposity and corrects insulin resistance in genetic and acquired models of obesity in mice [10]. Actually, these data seemed to challenge previous studies in humans, which reported elevated CT-1 levels in patients with hypertension and MS [11-13].

In this context, our study was the first to analyze serum CT-1 concentrations in patients undergoing coronary angiography. Consistent with the experimental background, but in discordance with studies in dysmetabolic patients [11-13], our results did not show associations between CT-1 and any component of the MS, while demonstrating an even inverse association of CT-1 with BMI and WC. We think that this apparent contradiction can be partially solved by analyzing carefully patient characteristics in the different studies. While Natal et al. determined serum CT-1 in "137 apparently healthy subjects free from clinically apparent atheroscle- rotic disease" [13], Pemberton et al. did so in 59 untreated and treated hypertensive patients without other specified comorbidities or complications [11], and Lopez et al. did so in 111 hypertensive patients without "clinical manifestations of ischaemic heart disease" [12], our study population consisted of patients who, even in the absence of already-established cardiac ischemic disease, did obtain a clinical indication for coronary angiography. Indeed, a significant proportion of our patients showed cardiovascular disease after examination (Table I), suggesting a long history of atherogenic morbidities. Therefore, as already proposed by Moreno-Aliaga et al. [10], it could be speculated that increased serum CT-1 could serve to counteract fat accumulation and metabolic-related complications in early obesity and MS, while this protective mechanism may fail in the late dysmetabolic patient, possibly due to a sort of exhaustion with a further negative impact at least on weight control. After all, insulin history has already indicated similar profiles in the same context: early hyperproduction to counteract hyperglycemia and late exhaustion which leads to type 2 diabetes.

Consistent with the lack of association between CT-1 and cardiovascular risk factors, we did not find any association between serum CT-1 and carotid or coronary artery disease. However, only studies including patients with a wider profile of CV risk could definitely clarify this issue.

In conclusion, our results, obtained in a population of candidates for coronary angiography, do not support any relationship between serum CT-1 and carotid or coronary artery disease, while they could help to solve the apparent contradiction raised by previous clinical and experimental studies on the metabolic roles of CT-1 in humans in vivo. 


\section{References}

1. Pennica D, King KL, Shaw KJ, et al. Expression cloning of cardiotrophin 1, a cytokine that induces cardiac myocyte hypertrophy. Proc Natl Acad Sci USA 1995; 92: 1142-6.

2. Pennica D, Swanson TA, Shaw KJ, et al. Human cardiotrophin-1: protein and gene structure, biological and binding activities, and chromosomal localization. Cytokine 1996; 8: 183-9.

3. González A, López B, Ravassa S, et al. Cardiotrophin-1 in hypertensive heart disease. Endocrine 2012; 42: 9-17.

4. Calabrò $P$, Limongelli $G$, Riegler $L$, et al. Novel insights into the role of cardiotrophin-1 in cardiovascular diseases. J Mol Cell Cardiol 2009; 46: 142-8.

5. Febbraio MA, Hiscock $N$, Sacchetti $M$, Fischer $C P$, Pedersen BK. Interleukin- 6 is a novel factor mediating glucose homeostasis during skeletal muscle contraction. Diabetes 2004; 53: 1643-8.

6. Watt MJ, Dzamko N, Thomas WG, et al. CNTF reverses obesity-induced insulin resistance by activating skeletal muscle AMPK. Nat Med 2006; 12: 541-8.

7. Kristiansen OP, Mandrup-Poulsen T. Interleukin- 6 and diabetes: the good, the bad, or the indifferent? Diabetes 2005; 54 Suppl 2: S114-24.

8. Tilg $\mathrm{H}$, Moschen AR. Adipocytokines: mediators linking adipose tissue, inflammation and immunity. Nat Rev Immunol 2006; 6: 772-83.

9. Stępień M, Wlazeł RN, Paradowski M, et al. Serum concentrations of adiponectin, leptin, resistin, ghrelin and insulin and their association with obesity indices in obese normo-and hypertensive patients - pilot study. Arch Med Sci 2012; 8: 431-6.

10. Moreno-Aliaga MJ, Pérez-Echarri N, Marcos-Gómez B, et al. Cardiotrophin-1 is a key regulator of glucose and lipid metabolism. Cell Metab 2011; 14: 242-53.

11. Pemberton CJ, Raudsepp SD, Yandle TG, Cameron VA, Richards AM. Plasma cardiotrophin-1 is elevated in human hypertension and stimulated by ventricular stretch. Cardiovasc Res 2005; 68: 109-17.

12. López B, González A, Lasarte JJ, et al. Is plasma cardiotrophin-1 a marker of hypertensive heart disease? J Hypertens 2005; 23: 625-32.

13. Natal C, Fortuño MA, Restituto $P$, et al. Cardiotrophin-1 is expressed in adipose tissue and upregulated in the metabolic syndrome. Am J Physiol Endocrinol Metab 2008; 294: E52-60.

14. Scanlon PJ, Faxon DP, Audet AM, et al. ACC/AHA guidelines for coronary angiography: executive summary and recommendations. A report of the American College of Cardiology/American Heart Association Task Force on Practice Guidelines (Committee on Coronary Angiography) developed in collaboration with the Society for Cardiac Angiography and Interventions. Circulation 1999; 99: 2345-57.

15. Third Report of the National Cholesterol Education Program (NCEP) Expert Panel on Detection, Evaluation, and Treatment of High Blood Cholesterol in Adults (Adult Treatment Panel III) final report. Circulation 2002; 106: 3143-421.

16. López B, González A, Querejeta R, Barba J, Díez J. Association of plasma cardiotrophin-1 with stage $C$ heart failure in hypertensive patients: potential diagnostic implications. J Hypertens 2009; 27: 418-24.

17. González A, Ravassa S, Loperena I, et al. Association of depressed cardiac gp130-mediated antiapoptotic pathways with stimulated cardiomyocyte apoptosis in hypertensive patients with heart failure. J Hypertens 2007; 25: 2148-57.
18. Janoschek R, Plum L, Koch L, et al. gp130 signaling in proopiomelanocortin neurons mediates the acute anorectic response to centrally applied ciliary neurotrophic factor. Proc Natl Acad Sci USA 2006; 103: 10707-12.

19. Febbraio MA. gp130 receptor ligands as potential therapeutic targets for obesity. J Clin Invest 2007; 117: 841-9.

20. Wojtaszewski JFP, Richter EA. Effects of acute exercise and training on insulin action and sensitivity: focus on molecular mechanisms in muscle. Essays Biochem 2006; 42: 31-46. 